\title{
Aligned Graphene Oxide Nanofillers: An Approach to Prepare Highly Thermally Conductive and Electrically Insulative Transparent Polymer Composites
}

\author{
Genlian Lin, Bin-Huan Xie, Juan Hu, Xiao Huang, and Guo-Jun Zhang \\ State Key Laboratory of High Performance Ceramics and Superfine Microstructures, Shanghai Institute of Ceramics, \\ Shanghai 200050, China
}

Correspondence should be addressed to Xiao Huang; xiaohuang@mail.sic.ac.cn and Guo-Jun Zhang; gjzhang@mail.sic.ac.cn

Received 13 April 2015; Accepted 27 May 2015

Academic Editor: Xuping Sun

Copyright (C) 2015 Genlian Lin et al. This is an open access article distributed under the Creative Commons Attribution License, which permits unrestricted use, distribution, and reproduction in any medium, provided the original work is properly cited.

Graphene oxide (GO)/polyvinyl alcohol composites with extremely high in-plane thermal conductivities are prepared by a simple tape casting process using water as process solvent. The in-plane thermal conductivity of the composite can reach $17.61 \mathrm{~W} / \mathrm{mK}$ at only $0.1 \mathrm{wt} \%$ GO loading, which is close to that of fully dense alumina. The excellent thermal conducting ability, the unique twodimensional morphology, and the all-wet handling of the GO fillers as well as the high orientation of the fillers in the polymer matrix all contribute to the high thermal conductivities achieved. Meanwhile, the composites show good electrical insulation property and decent transparency.

\section{Introduction}

The intrinsic thermal conductivities of polymers are generally much lower than those of other materials. Introducing thermal conductive fillers is one convenient and effective way to enhance the thermal conducting abilities of polymers [1]. However, until now, in order to increase polymer's thermal conductivity significantly, high filler loading (more than $30 \mathrm{vol} \%$ ) is always required $[1,2]$, which often leads to other unwanted issues, such as high cost, difficulties in processing, brittleness, and weight increase. Achieving high thermal conductivities with quite low filler loadings is highly expected and still remains as a challenge.

Various thermal conductive fillers have been incorporated into polymer matrix to increase their thermal conductivities, such as metals, ceramics particles, nanoclays, and carbon-based materials [1]. Among all potential thermal conductive fillers, nanoscaled fillers, especially one-dimensional nanofillers, such as carbon nanotubes (CNTs), have been favored for the past decade due to their extremely low percolation threshold comparing to other fillers [3]. However, although CNTs as well as other one-dimensional nanofillers can form percolating network at very low concentrations as evidenced by electrical conductivities above the percolation threshold, their abilities to enhance thermal conductivity are rather disappointing and much lower than the theoretical predictions $[1,3]$.

Graphene is an amazing material. Since its discovery, graphene has attracted enormous interest both in scientific research and in industry. It has been reported that the percolation threshold of graphene/polystyrene composites is as low as $0.1 \mathrm{vol} \%$ [4]. The small percolation threshold, the ultrahigh in-plane thermal conductivity $(\sim 5000 \mathrm{~W} / \mathrm{mK})$, and its two-dimensional structure make graphene one of the most potential thermal conductive filler candidates. Xie et al. theoretically studied graphene nanosheets and CNTs as secondary fillers for polymer composites and suggested that two-dimensional graphene nanosheets are more effective than one-dimensional CNT in enhancing the thermal conductivity [5].

In this work, graphene oxide (GO)/polyvinyl alcohol (PVA) composites are prepared by a simple environmental friendly process using water as process solvent. GO is selected because it bears abundant hydroxyl, carbonyl, and carboxyl groups [6] leading to excellent dispersibility in aqueous solution and stronger interactions (H-bonding) with PVA. Low dimensional nanofillers with large aspect ratio, such as CNTs and graphene, often show obvious anisotropy in their 
thermal conducting abilities. By controlling the orientation of the nanofillers, higher thermal conductivities in desired direction can be achieved [7-9]. GO fillers can be easily aligned during a so-called tape casting process, which leads to superior in-plane thermal conducting abilities. The inplane thermal conductivity of the PVA composite can reach $17.61 \mathrm{~W} / \mathrm{mK}$ at only $0.1 \mathrm{wt} \% \mathrm{GO}$ loading. And that value can further increase to $24.63 \mathrm{~W} / \mathrm{mK}$ at $5 \mathrm{wt} \%$ loading. Meanwhile, the composites show good electrical insulation property and decent transparency.

\section{Materials and Methods}

Graphite oxide was synthesized from graphite by the Hummers method [12]. The dispersions of individual GO sheets in water $\left(7 \mathrm{mg} \mathrm{mL}^{-1}\right)$ were prepared by ultrasonication according to the literature $[13,14]$. PVA (MW: $1750 \pm 50)$ was obtained from Sinopharm Chemical Reagent Co., Shanghai, China, used as received.

Certain amount of GO aqueous dispersion was mixed with a $5 \mathrm{wt} \%$ PVA aqueous solution. The mixture was first magnetically stirred at room temperature for $30 \mathrm{~min}$ and then sonicated for an additional $30 \mathrm{~min}$ to obtain uniform dispersions. Then the GO/PVA nanocomposite films were prepared by tape casting, as previously reported [1].

XRD profiles were recorded using a Rigaku X-ray diffractometer (D/Max-2250 V, Japan) with $\mathrm{Cu} \mathrm{K} \alpha$ radiation. SEM images were taken on a Hitachi S-4800 scanning electron microscope. Measurements of the cross-plane and in-plane thermal conductivities of the samples (cross-plane: $10 \mathrm{~mm} \times$ $10 \mathrm{~mm}$, thickness $0.1 \sim 2.5 \mathrm{~mm}$; in-plane: diameter $25.4 \mathrm{~mm}$, thickness $0.1 \sim 0.5 \mathrm{~mm}$ ) were carried out at room temperature using a laser-flash diffusivity instrument (Nano-FlashApparatus, LFA 447, NETZSCH) via two different modes. The thermal conductivity data reported were the average of three individual measurements. SThM (scanning thermal microscope) images were taken on a commercial atomic force microscope (SPA 400, SPI3800N, Seiko Inc., Japan) equipped with a customer-built thermal scanning probe to give out surface topography image and thermal transporting information simultaneously. The optical transmittances of the composite films were measured on a Shimadzu UV-3101PC spectrophotometer. Sonication of GO dispersions was performed by a Sonics Vibra Cell sonicator. Electrical resistivity was measured on a Physical Properties Measurement system (PPMS-9, Quantum Design Co.) at room temperature.

\section{Results and Discussion}

SEM images of graphite oxide and GO are shown in Figures 1(a) and 1(b). After sonication, graphite oxide is exfoliated well into GO nanosheets, and no agglomerated particles can be observed. XRD profiles of graphite, graphite oxide, GO and GO/PVA composite (10 wt $\%$ GO) are illustrated in Figure 1(c). Very sharp and strong diffraction peak around $26^{\circ}$ is characteristic of graphite. This peak shifts to around $12^{\circ}$ and becomes much broader after oxidation, which is an indication of successful conversion from graphite to graphite oxide. The increasing $d$-spacing is due to water molecule intercalation [5]. Sonication of graphite oxide in water is a very common and efficient way to exfoliate graphite oxide to monolayered or few-layered GO $[7,8]$. The even broader peak around $10^{\circ}$ in GO can be attributed to the fact that GO nanosheets easily restack upon drying. The peak around $20^{\circ}$ in GO/PVA composite is coming from PVA, while the shoulder around $15^{\circ}$ is possibly due to small degree of GO nanosheet restacking at this high loading. These results indicate that exfoliated GO nanosheets are well dispersed in PVA matrix. Photographs of GO/PVA composite films at 3 and $5 \mathrm{wt} \%$ GO loadings, which are shown in Figure 2(a), are direct observations of homogeneous distribution of GO in the polymer matrix.

The cross section of the $3 \mathrm{wt} \%$ GO/PVA composite film is observed by SEM (Figure 2(b)). It can be clearly seen that GO nanosheets are dispersed nicely in the polymeric matrix and no big particles or agglomerates can be observed. Careful examination on the image indicates that the GO nanosheets are well aligned along the tape casting direction.

The thermal conductivities of GO/PVA composite films at various filler loadings are summarized in Figure 3(a). The thermal conductivities along the film plane increase significantly due to the effective alignment of GO nanosheets during tape casting process $[2,6-8]$. The in-plane thermal conductivity of the composite is as high as $17.61 \mathrm{~W} / \mathrm{mK}$ at $0.1 \mathrm{wt} \%$ filler loading and can reach a value of $24.63 \mathrm{~W} / \mathrm{mK}$ at $5 \mathrm{wt} \%$ loading. The latter value is close to the thermal conductivity of fully dense alumina [15], which is one of the most widely used electronic packaging substrate materials. It seems that the thermal conductive pathways can be formed at $0.1 \mathrm{wt} \%$ GO loadings in GO/PVA composite. Apparently, the nanosheet morphology and the alignment along the planar direction both contribute to this low percolation threshold (Figure 4(a)).

SThM is a powerful scanning probe microscope technique. In SThM, the interaction between the tip and sample surface is related to the heat flux. Different temperature/thermal conductivity on the sample surface constructs the image. Thus SThM can reveal the microstructure and the thermal transporting ability of our composites at nanoscale simultaneously (Figures 3(b)-3(d)). The pixel brightness in the thermal images (Figures 3(c) and 3(d)) is an indication of thermal transport. The brighter the pixel, the faster the heat flux (i.e., large thermal conductivity) in that region. The thermal image of GO/PVA composite shows quite homogeneous brightness, which indicates that GO disperses uniformly in the PVA matrix and a continuous thermal conductive pathway is formed. As a comparison, the SThM image of micron-sized graphite oxide/PVA composite is also shown in Figure $3(\mathrm{~d})$. The obvious bright and dark contrast indicates the less well-formed thermal conductive pathways, which results in a much lower thermal conductivity comparing to its GO opponents.

UV-vis spectra of GO/PVA composite films are shown in Figure 3(e). Because it is impossible to control the film thickness to be exactly the same, the transmittance data in Figure 3(e) are normalized based on $10 \mu \mathrm{m}$ film thickness. The pure PVA film has a transmittance greater than $98 \%$. 


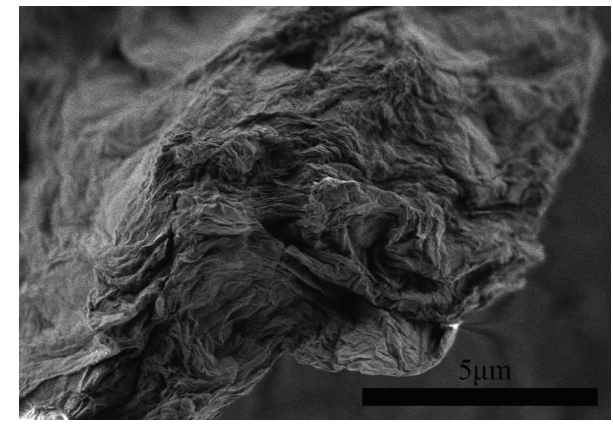

(a)

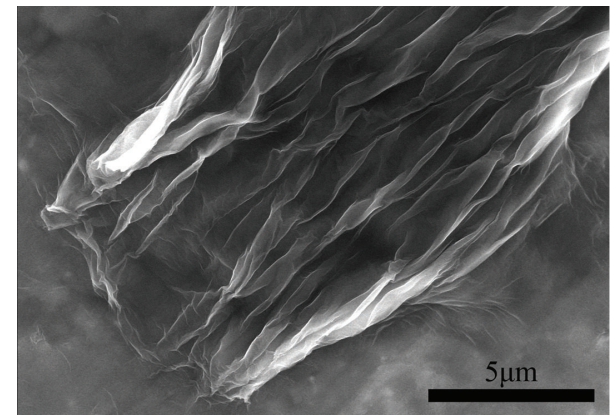

(b)

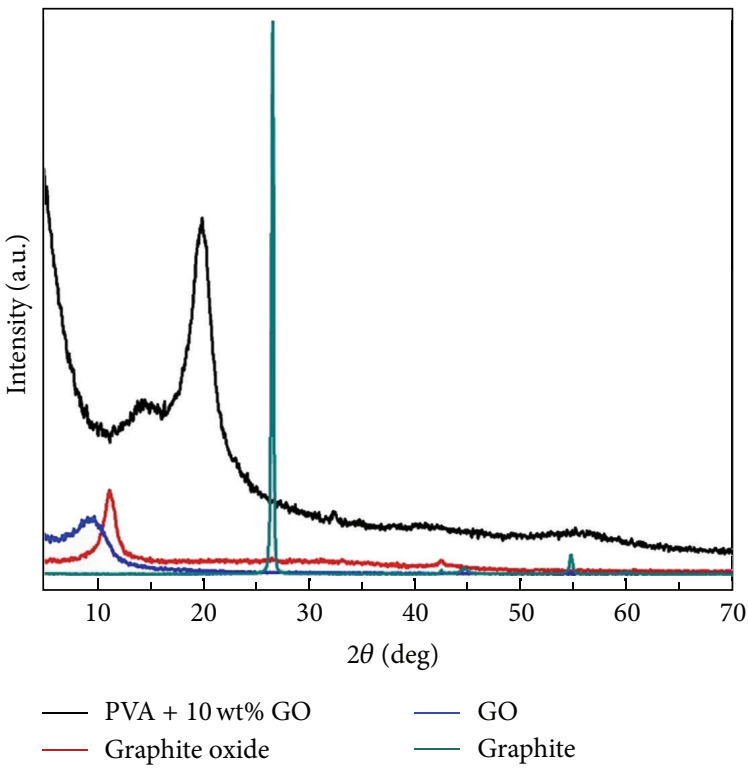

(c)

FIGURE 1: SEM images of (a) graphite oxide and (b) graphene oxide (GO). (c) XRD profiles of graphite, graphite oxide, GO and GO/PVA composite (10 wt\% GO).

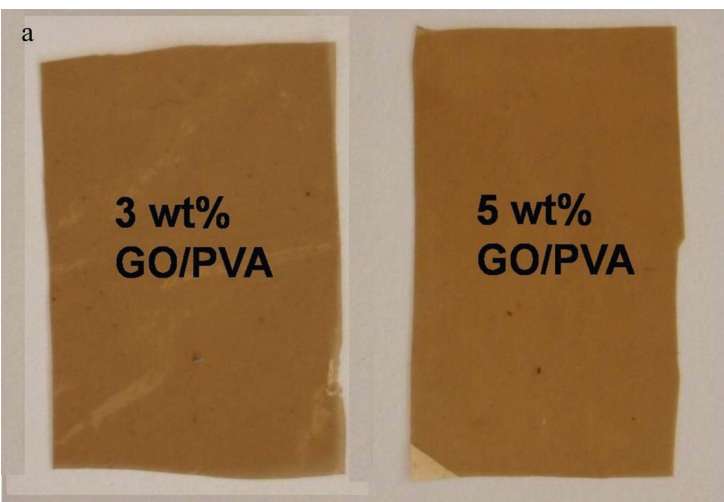

(a)

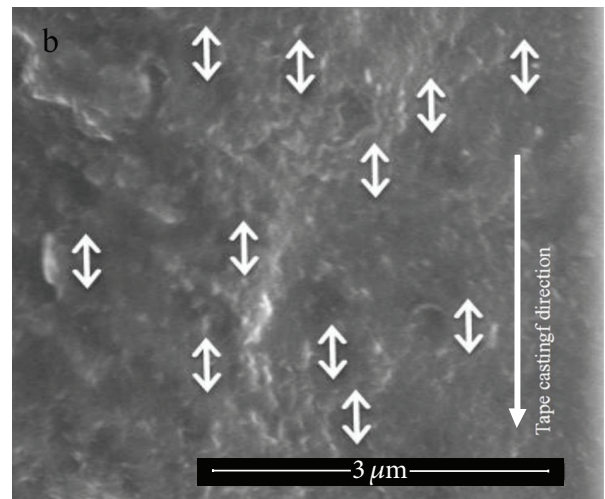

(b)

Figure 2: (a) Photographs of GO/PVA composite films by tape casting at 3 and $5 \mathrm{wt} \%$ filler loadings, (b) SEM image of the cross section of GO/PVA composite (3 wt\% loading). 


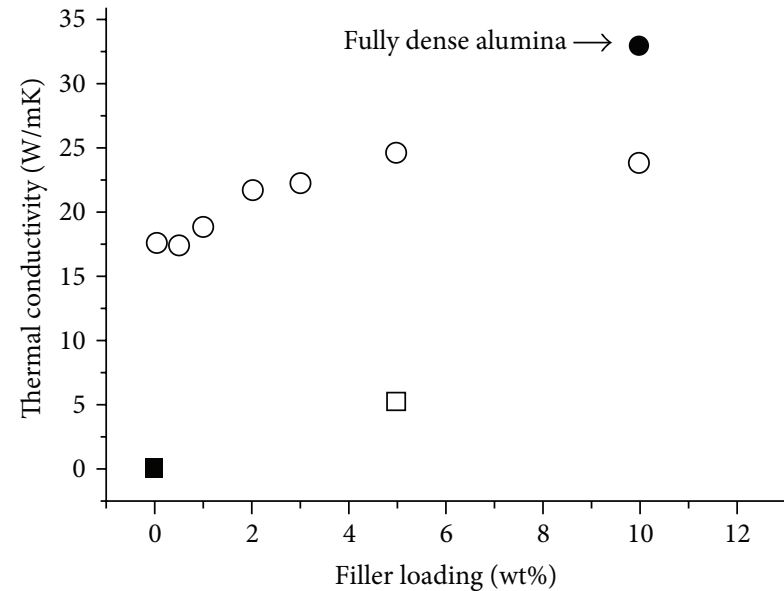

(a)

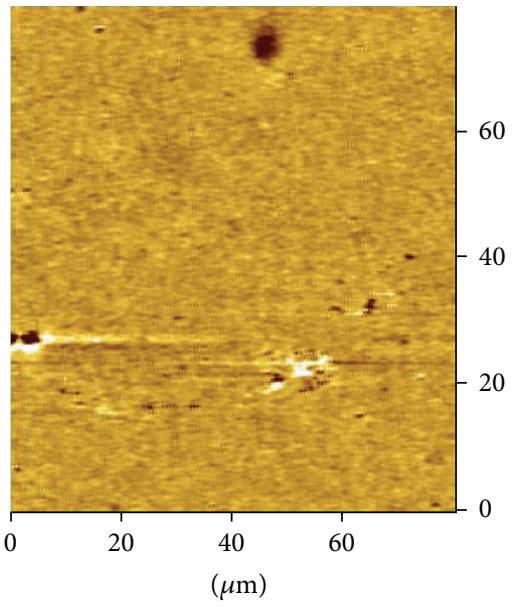

(c)

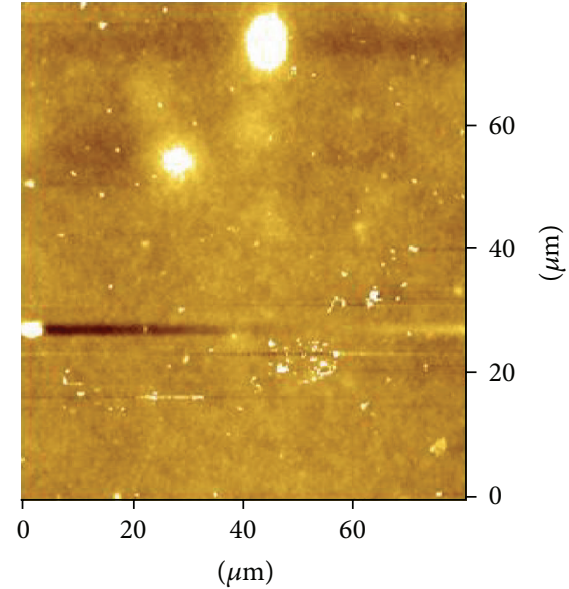

(b)

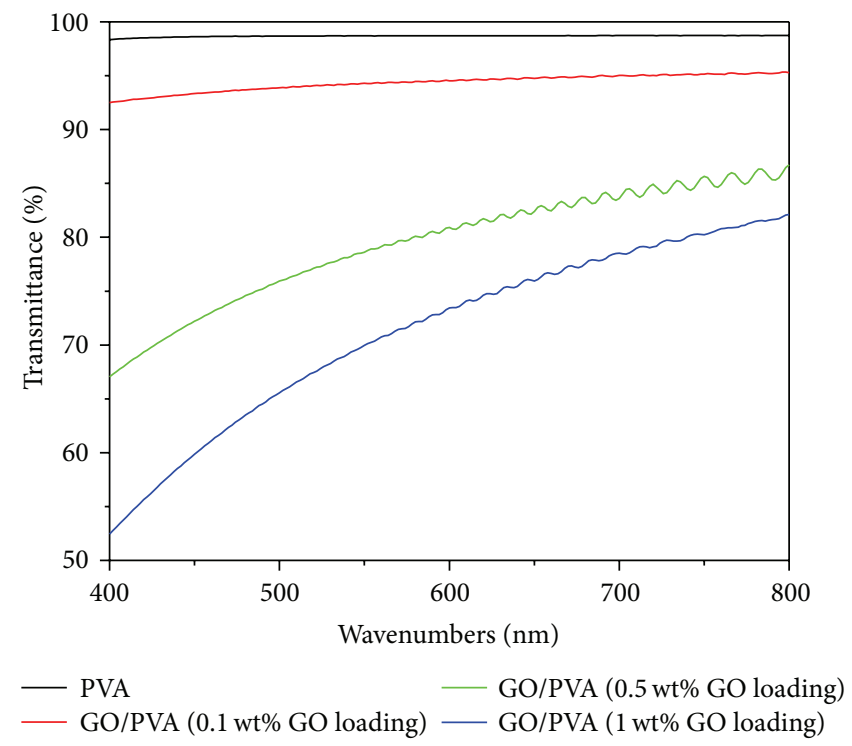

(e)

Figure 3: (a) Thermal conductivities of GO/PVA composites at different filler loads ( $\bigcirc$, in-plane), pure PVA ( $\square$ ), graphite oxide/PVA composite at $5 \mathrm{wt} \%$ filler loading ( $\square$, in-plane) and fully dense alumina (๑); (b) topography and (c) thermal image of GO/PVA (5 wt\% GO loading; $25 \mathrm{~W} / \mathrm{mK}$ ); (d) thermal image of micron-sized graphite oxide/PVA composite ( $5 \mathrm{wt} \%$ filler loading; $5 \mathrm{~W} / \mathrm{mK}$ ); and (e) the transmittance of GO/PVA composites by tape casting with different loadings. 


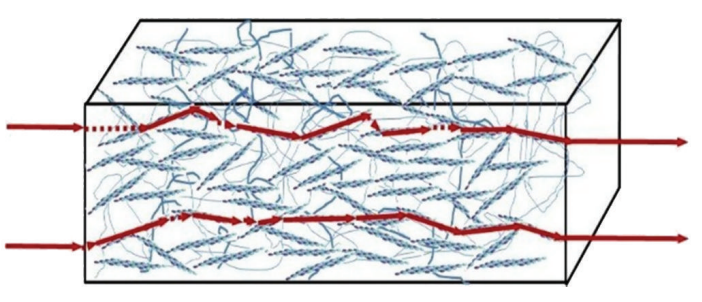

(a)

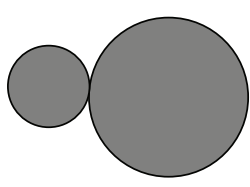

One-dimensional and particulate fillers

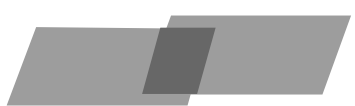

Two-dimensional GO fillers

(b)

FIGURE 4: Illustration of (a) polymer composite with fillers forming thermal conductive pathways over percolation threshold, the red lines are indication of heat flow, (b) possible contact between fillers with different morphologies.

TABLE 1: Summary of the in-plane thermal conductivities of aligned nanofiller/polymer composites*.

\begin{tabular}{lccc}
\hline Material & $\begin{array}{c}\text { Filler } \\
\text { loading }\end{array}$ & $\begin{array}{c}\text { In-plane } \\
\text { thermal } \\
\text { conductivity } \\
(\mathrm{W} / \mathrm{mK})\end{array}$ & Ref. \\
\hline Aligned CNT paper & $100 \%$ & $\sim 42$ & {$[8]$} \\
\hline Aligned CNT/epoxy composite & $50 \mathrm{vol} \%$ & $\sim 7$ & {$[8]$} \\
\hline Aligned CNT/PVA composite & $10 \mathrm{wt} \%$ & $\sim 0.5$ & {$[10]$} \\
\hline Aligned graphite/EPDM & 31.6 & 7.14 & {$[11]$} \\
\hline Aligned carbon fiber/EPDM & $15 \mathrm{vol} \%$ & 1.23 & {$[11]$} \\
\hline Aligned CNT/EPDM & $15 \mathrm{vol} \%$ & 1.15 & {$[11]$} \\
\hline Aligned GR/epoxy & $1 \mathrm{vol} \%$ & $\sim 0.6$ & {$[7]$} \\
\hline Aligned BNNS/nanofibrillated & $5 \mathrm{wt} \%$ & 26.2 & {$[9]$} \\
cellulose & $50 \mathrm{wt} \%$ & 145.7 & \\
\hline Aligned BN microplates/PVA & $1 \mathrm{wt} \%$ & 1.45 & {$[2]$} \\
\hline Aligned GO/PVA & $10 \mathrm{wt} \%$ & 3.92 & \\
\hline
\end{tabular}

*EPDM: ethylene-propylene-diene copolymer; BNNS: boron nitride nanosheet; and $\mathrm{BN}$ : boron nitride.

The GO/PVA composite at $0.1 \mathrm{wt} \%$ GO loading show a transmittance over $92 \%$, while its in-plane thermal conductivity is as high as $17.61 \mathrm{~W} / \mathrm{mK}$. When we further increase the filler loadings, the transmittance of the films decreases quickly as predicted. The vibration observed in the spectra is due to the interference of light, which is commonly observed in transparent thin films [16].

Electrical resistance of GO/PVA composite is measured to be $5.8 \times 10^{5} \Omega \cdot \mathrm{m}$ (in-plane), which is close to that value of pure PVA $\left(3.1 \sim 3.8 \times 10^{5} \Omega \cdot \mathrm{m}\right)$. The cross-plane electrical resistance is too large to be measured by the instrument used. Obviously, introducing GO into PVA matrix does not change its electrical insulation nature as expected.

The thermal conductivities of our GO/PVA composites are obviously much higher than those in previously reported work, including those in which the fillers were also oriented. Those data are listed in Table 1 for comparison. It has been found that films made of aligned CNTs, the thermal conductivities along the oriented direction, are an order of magnitude greater than that of unoriented materials and the cross-plane value of the same material [8]. However, when CNTs are introduced into polymer composites, even though they can still be highly oriented, the thermal conductivities along the orientation direction are much lower than expected [8]. The poor performance of CNT in polymer matrix is often attributed to the high interfacial thermal resistance and high tube-tube contact resistance $[1,3]$. However, besides the factors such as interfacial thermal resistance, fillerfiller contact resistance, fillers' dispersibility, and percolation threshold, the contact (overlapping) area of fillers should also be a very important factor for heat flow. A theoretical study done by Shenogina et al. shows that different from electrical conductivity, thermal conductivity of the polymeric composites can be significantly enhanced only when the fillers are in contact. This is because the ratio of thermal conductivities between filler and matrix is much smaller than that of electron conductivities [17].

The degree of overlapping on the interfacial area is strongly affected by filler's morphology. It increases as the fillers deviate from perfect spherical morphology, that is, aspect ratio [18]. As demonstrated in Figure 4(b), the contact between one-dimensional and particulate fillers is point-topoint, while the contact between two-dimensional fillers can be face-to-face (the flat illustration of GO nanosheets is ideal). Apparently, two-dimensional fillers can provide much larger overlapping area than the other two, which might make them more promising thermal conductive fillers and be one of the reasons for such high thermal conductivity obtained in this work.

It is well known that GO or graphene nanosheets tend to restack and form wrinkled structure especially upon drying. Restacking of GO nanosheets is possible to disfavor the formation of percolated network; that is, percolation threshold increases, while wrinkling will reduce the effective aspect ratio of the filler. In our experiments, the GO is always dispersed in water during the whole process to avoid restacking and wrinkling, which may be another important contributor to the high thermal conductivities observed in the composites.

\section{Conclusions}

In summary, GO nanosheets are introduced into PVA matrix to improve polymer's thermal conductivity by a simple 
environmental friendly process in this work. GO/PVA composite films are prepared by tape casting, which can easily make the GO nanosheets well align along the film surface. Anisotropic thermal conductivities are observed due to the shape and orientation of those nanosheets. Very high inplane thermal conductivities of GO/PVA composites can be achieved at very low filler loadings. In-plane thermal conductivity of $17.61 \mathrm{~W} / \mathrm{mK}$ can be reached at only $0.1 \mathrm{wt} \% \mathrm{GO}$ loading, which is about 100 times higher than the PVA matrix. And that value can further increase to $24.63 \mathrm{~W} / \mathrm{mK}$ at $5 \mathrm{wt} \%$ loading, which is close to the thermal conductivity of fully dense alumina. The unique 2D morphology of GO, which can provide largest overlapping area between filler particles, may be an important reason for its superior performance in promoting polymer thermal conducting abilities to other fillers, especially CNT. The all-wet handle of GO prevents GO nanosheets from restacking and forming wrinkled structure is another key contributor to the high thermal conductivities obtained.

Besides being highly thermally conductive, the GO/PVA composite at $0.1 \mathrm{wt} \%$ loading is electrical insulative and transparent in visible region, which makes this composite very appealing to many applications.

\section{Conflict of Interests}

The authors declare that there is no conflict of interests regarding the publication of this paper.

\section{Authors' Contribution}

Genlian Lin and Bin-Huan Xie contribute equally to this work.

\section{Acknowledgments}

Financial supports from the Chinese Academy of Sciences (Hundred Talents Program) and the National Basic Research Program (Project no. 2012CB720904) are gratefully acknowledged. The authors thank Professor Hua-Rong Zeng of the Shanghai Institute of Ceramics, for his generous help in obtaining the SThM images.

\section{References}

[1] Z. Han and A. Fina, "Thermal conductivity of carbon nanotubes and their polymer nanocomposites: a review," Progress in Polymer Science, vol. 36, no. 7, pp. 914-944, 2011.

[2] B.-H. Xie, X. Huang, and G.-J. Zhang, "High thermal conductive polyvinyl alcohol composites with hexagonal boron nitride microplatelets as fillers," Composites Science and Technology, vol. 85, pp. 98-103, 2013.

[3] S. T. Huxtable, D. G. Cahill, S. Shenogin et al., "Interfacial heat flow in carbon nanotube suspensions," Nature Materials, vol. 2, no. 11, pp. 731-734, 2003.

[4] S. Stankovich, D. A. Dikin, G. H. B. Dommett et al., "Graphenebased composite materials," Nature, vol. 442, no. 7100, pp. 282286, 2006.
[5] S. H. Xie, Y. Y. Liu, and J. Y. Li, "Comparison of the effective conductivity between composites reinforced by graphene nanosheets and carbon nanotubes," Applied Physics Letters, vol. 92, no. 24, Article ID 243121, 2008.

[6] A. Buchsteiner, A. Lerf, and J. Pieper, "Water dynamics in graphite oxide investigated with neutron scattering," The Journal of Physical Chemistry B, vol. 110, no. 45, pp. 22328-22338, 2006.

[7] H. Yan, Y. Tang, W. Long, and Y. Li, "Enhanced thermal conductivity in polymer composites with aligned graphene nanosheets," Journal of Materials Science, vol. 49, no. 15, pp. 5256-5264, 2014.

[8] P. Gonnet, Z. Liang, E. S. Choi et al., "Thermal conductivity of magnetically aligned carbon nanotube buckypapers and nanocomposites," Current Applied Physics, vol. 6, no. 1, pp. 119$122,2006$.

[9] H. Zhu, Y. Li, Z. Fang et al., "Highly thermally conductive papers with percolative layered boron nitride nanosheets," ACS Nano, vol. 8, no. 4, pp. 3606-3613, 2014.

[10] W.-L. Song, W. Wang, L. M. Veca et al., "Polymer/carbon nanocomposites for enhanced thermal transport propertiescarbon nanotubes versus graphene sheets as nanoscale fillers," Journal of Materials Chemistry, vol. 22, no. 33, pp. 17133-17139, 2012.

[11] J.-H. Kim and G.-H. Kim, "Effect of orientation and content of carbon based fillers on thermal conductivity of ethylenepropylene-diene/filler composites," Journal of Applied Polymer Science, vol. 131, no. 21, 2014.

[12] W. S. Hummers Jr. and R. E. Offeman, "Preparation of graphitic oxide," Journal of the American Chemical Society, vol. 80, no. 6, p. 1339, 1958.

[13] H. A. Becerril, J. Mao, Z. Liu, R. M. Stoltenberg, Z. Bao, and Y. Chen, "Evaluation of solution-processed reduced graphene oxide films as transparent conductors," ACS Nano, vol. 2, no. 3, pp. 463-470, 2008.

[14] D. A. Dikin, S. Stankovich, E. J. Zimney et al., "Preparation and characterization of graphene oxide paper," Nature, vol. 448, no. 7152, pp. 457-460, 2007.

[15] W. Pabst and E. Gregorová, "New developments in materials science research," in Effective Thermal and Thermoelastic Properties of Alumina, Zirconia and Alumina-Zirconia Composite Ceramics, B. M. Caruta, Ed., pp. 77-138, Nova Science Publishers, New York, NY, USA, 2007.

[16] J. C. Manifacier, J. Gasiot, and J. P. Fillard, "A simple method for the determination of the optical constants $\mathrm{n}, \mathrm{k}$ and the thickness of a weakly absorbing thin film," Journal of Physics E: Scientific Instruments, vol. 9, no. 11, pp. 1002-1004, 1976.

[17] N. Shenogina, S. Shenogin, L. Xue, and P. Keblinski, "On the lack of thermal percolation in carbon nanotube composites," Applied Physics Letters, vol. 87, Article ID 133106, 2005.

[18] W. Xu, W. Chen, H. Chen, X. Tian, and H. Zhao, "Determination of overlapping degree of interfacial layers around polydisperse ellipsoidal particles in particulate composites," Physica A, vol. 399, pp. 126-136, 2014. 

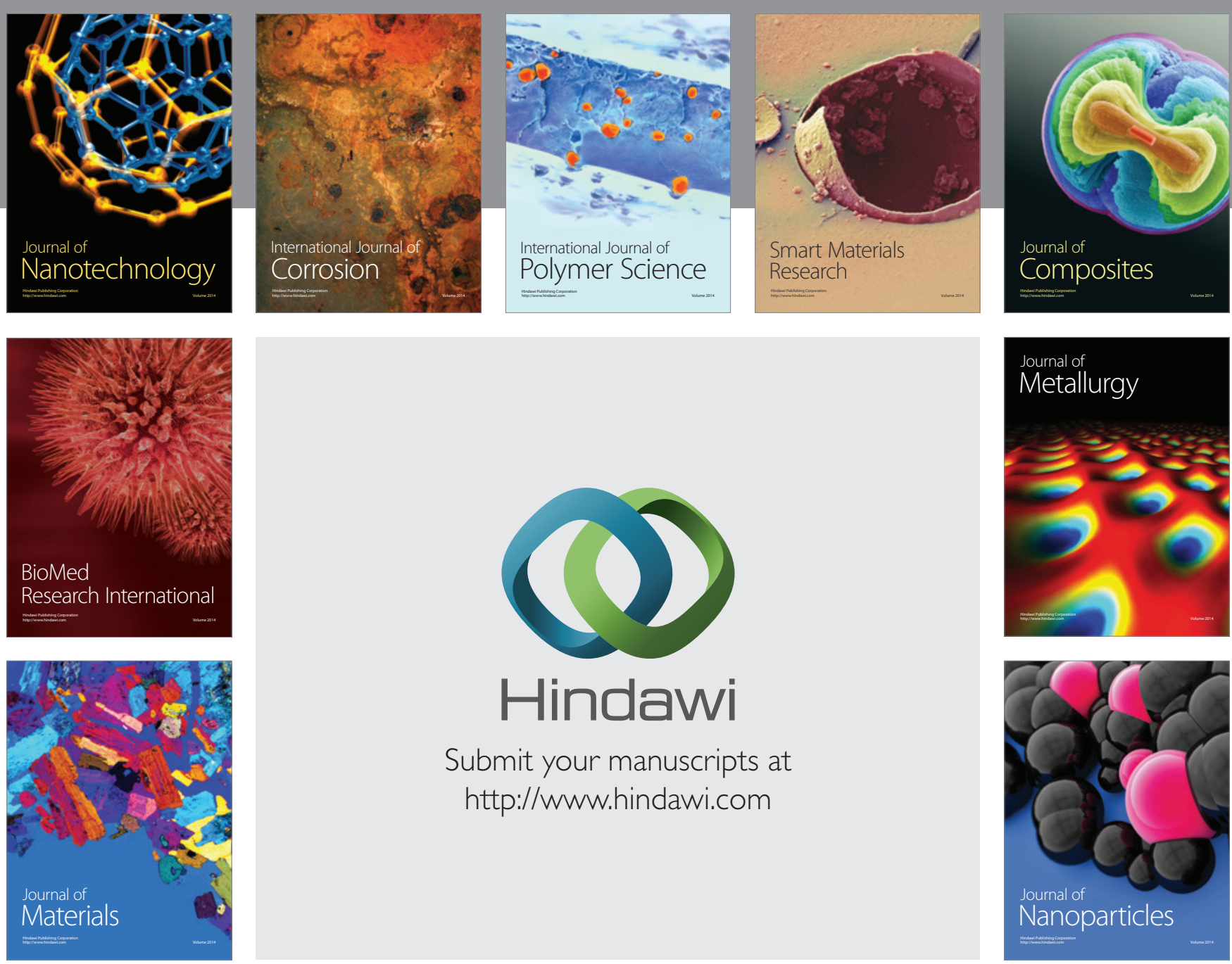

Submit your manuscripts at http://www.hindawi.com
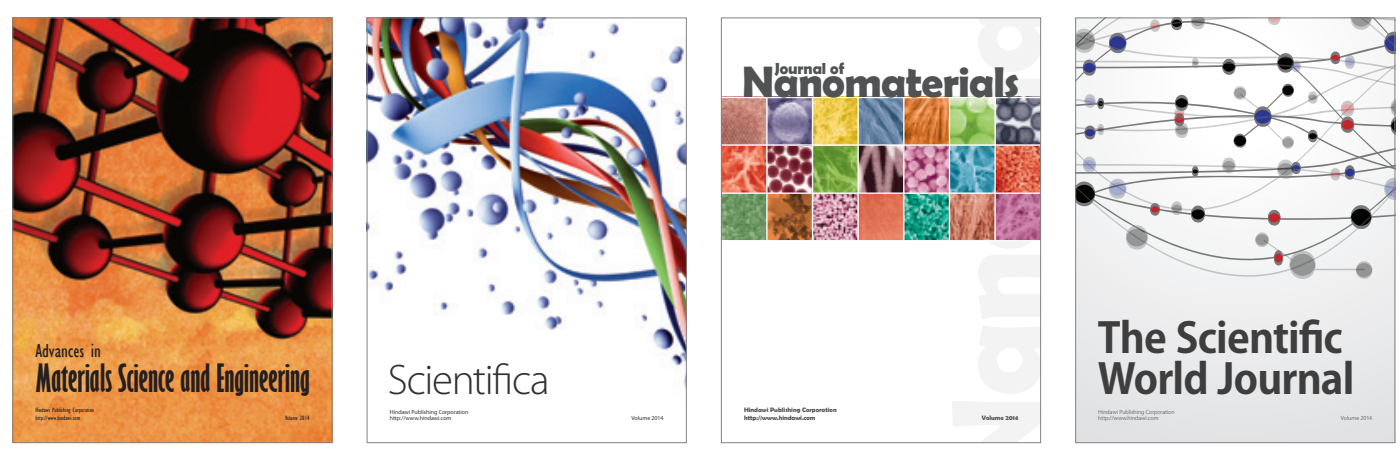

\section{The Scientific World Journal}
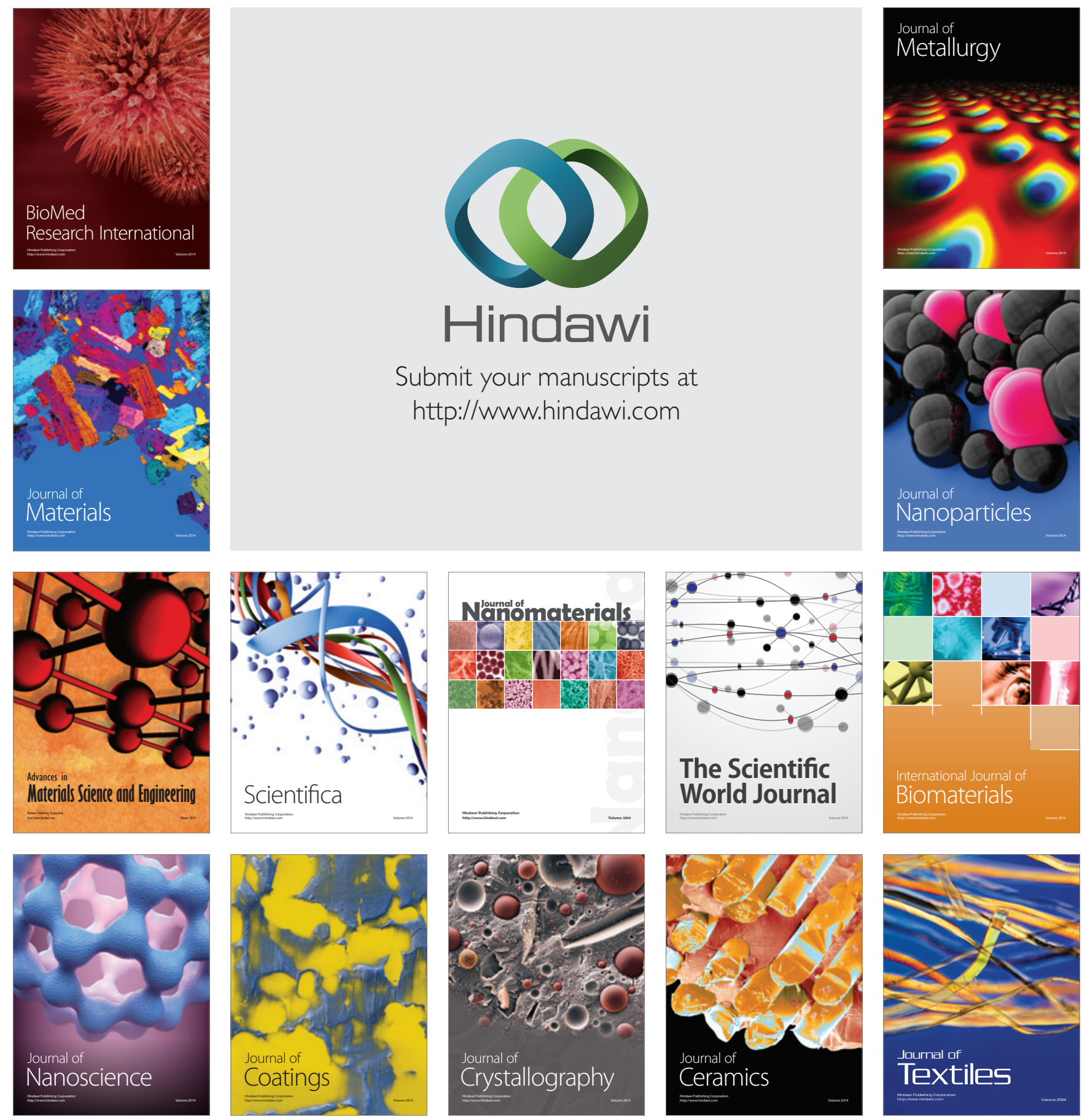